\title{
Geographical variation in cancer patient survival in Finland: chance, confounding, or effect of treatment?
}

Sakari Karjalainen

\begin{abstract}
Study objective-The aim was to determine whether survival of cancer patients in Finland varies with their place of residence, and if so, what proportion of the variation might be due to health services rather than to confounding variables.

Design-Patients with breast and prostatic cancer diagnosed in Finland between 1970 and 1981 were classified by place of residence (from 21 hospital districts), and area specific 5 year relative survival rates were estimated.

Setting-This was a population based survey of the whole of Finland.

Patients-16 754 cases of breast cancer and 9483 cases of prostatic cancer were identified. Of these, $0.5 \%$ of breast cancers and $4 \cdot 1 \%$ of prostatic cancers were excluded because diagnosis was based only on necropsy findings or because the only information available was from the death certificate.
\end{abstract}

Measurements and main results-There was a large variation in rates, ranging from $59 \%$ to $76 \%$ for breast cancer, and from $30 \%$ to $65 \%$ for prostatic cancer. However, after accounting for age of patient and extent of disease, the standardised differences for prostatic cancer closely followed random distribution, indicating equal results of treatment in different areas. For breast cancer there was more variation than expected by chance and patients resident in any of the university central hospital districts with modern radiotherapy equipment survived better than other patients.

Conclusions-There is little indication that large variations in crude mortality rates from these two cancers in different parts of Finland are due to inequalities of medical care, though a small effect on breast cancer survival which might be care related was shown.

Regional differences in the allocation of health services resources, or their use, or regional variations in health status, are used to indicate equity, one of the dimensions of quality of health care. ${ }^{1-3}$ As a rule in clinical oncology the outcome of the treatment indicates quality, and is given in terms of survival. The survival of a cancer patient depends on the characteristics of the patient, on the biology of the disease, and in part on the quality of the medical services. Some part of the differences in the survival rates of cancer patients is due only to random variation.
The purpose of this paper was to determine (1) whether the survival of cancer patients in Finland varies with their place of residence, and (2) if such variation is present, what proportion of the difference can be accounted for by chance, or by confounding from geographical differences in patient or disease characteristics, as opposed to possible variations in the health services. The methodology developed and described earlier ${ }^{4}$ was applied to breast and prostate cancer, which are the most frequent malignant tumours with fairly good prognosis in Finland.

The approach was population based. All cancer patients residing in an area served by one of the Finnish central hospitals were included and classified by their place of residence, irrespective of whether they were treated in hospitals of their own area, somewhere else, or not at all. The patients were followed for several years after their course of treatment was completed, and five year survival was estimated.

\section{Methods}

PATIENTS AND HEALTH CARE FACILITIES

The Finnish health services are based on financially autonomous municipalities each having the primary health services. The communes have the right to cooperate and several communes form a central hospital district (later: hospital district). ${ }^{5}$ The patients were classified by their place of residence into 21 groups corresponding to the 21 hospital districts. Most of the patients enter into the health services through municipal health centres or through private practitioners who refer the patient for diagnostic confirmation, usually to one of the central hospitals.

It is at the district level, where the cancer diagnostic facilities and treatment are centralised, that the area differences exist. During the 1970s, only eight of the total of 21 hospital districts had radiotherapy units giving external beam therapy. Cancer surgery was therefore practised in all of the 21 hospital districts, whereas radiotherapy was centralised in four of the five university hospitals and four other hospitals. These eight radiotherapy units were thus responsible for a larger catchment area than their own hospital district. The five medical schools of the country are closely related to the corresponding university hospitals. These were assumed to represent the highest level of medical expertise in the country.

All breast and prostatic cancer patients diagnosed in Finland in the period 1970-1981 and reported to the Finnish Cancer Registry were included in this study. The Finnish Cancer Registry is nationwide and population based. 
Hospitals, pathology laboratories, and private practitioners report every cancer case that comes to their attention. In addition, annual checks are made against the registry files of all death certificates issued in the country. Accordingly, the registry acquires information on deaths from cancer, whether diagnosed before or after death, and from deaths of cancer patients from causes other than cancer. Various checks have indicated that the registry files can be considered practically complete for cases of cancer diagnosed in Finland ${ }^{6}$ and that also the follow up of the cancer patients is virtually complete. ${ }^{7}$

There were 16754 breast cancer (ICD9 174) and 9483 prostatic cancer (ICD9 185) cases diagnosed in Finland in 1970-1981. Of the original material, $0.5 \%$ of breast cancers and $4.1 \%$ of prostatic cancers were excluded because the diagnosis was based on autopsy or because the information at the registry originated from the death certificate only. The proportion of excluded cases varied from nil to $1.0 \%$ in breast cancer, and from $1.0 \%$ to $7 \cdot 3 \%$ in prostatic cancer. The classification of the patients into 21 hospital districts resulted in rather small numbers of cases for the smallest districts. Only those hospital districts which produced more than 20 patients for each specific analysis were included.

\section{ANALYSES}

Date of diagnosis reported by hospitals was used as a starting point for the follow up. Survival of the patients was estimated by the life table method. ${ }^{8}$ The crude five year survival rates for area $j\left(P_{j}\right)$ were corrected for other causes of death by dividing the observed rates by those expected in a similar population by age and sex but free of the cancer under study. ${ }^{9}$ These corrected rates, called the relative survival rates $\left(\mathbf{R}_{\mathfrak{j}}\right)$, were also corrected for heterogeneity in patient withdrawal. ${ }^{10}$ The traditional measure of the statistical precision of the survival rate is the standard error $\left(S\left[P_{j}\right]\right)$. This was estimated by Greenwood's formula, ${ }^{8}$ which yields directly the standard error or the relative survival rate $\left(S\left[R_{\mathfrak{j}}\right]\right)$. Even if the quality of treatment of the cancer patients was the same, ie, the theoretical survival rates of patients by hospital districts were identical, the observed rates would not be identical because of a random variation and confounding effects.

Confounding occurs if a geographically varying factor is related to survival. Selected confounders related to disease and the patient were considered. Confounding due to primary site of the tumour and extent of the disease was controlled for by analysing the survival separately for selected primary sites (breast and prostate) and for localised and non-localised tumours. The classification of stage was based on the stage at time of diagnosis reported by the clinician who had made the diagnosis. The confounding effect of age was eliminated in two stages: relative survival rates eliminate the effect of age related general mortality, and indirect adjustment ${ }^{11}$ for age was used to eliminate the cancer related differences by age in survival rates.

The indicator for true equality in the survival rates is the distribution of standardised differences $\left(\mathbf{D}_{\mathbf{j}}\right)$ :

$$
\mathbf{D}_{\mathbf{j}}=\left(\mathbf{R}_{\mathbf{j}}-\mathrm{E}\left(\mathbf{R}_{\mathbf{j}}\right)\right) / \mathbf{S}\left(\mathbf{R}_{\mathfrak{j}}\right)
$$

where $E\left(R_{j}\right)$ is the expected relative survival rate, which is based on the total patient population over all hospital districts. The methodology has been described and discussed in detail in the article by Hakama et al. ${ }^{4}$

The distribution of the standardised differences should follow approximately the normal probability law, with mean $=0$ and standard deviation $=1$, on the assumption of equal theoretical survival in each of the hospital districts and only chance affecting the variation. Because there are 21 hospital districts in Finland, the expected number of standardised survival differences exceeding 2 in absolute value is one. Systematic variation will express itself in wider
Number, mean age, 5 year observed survival rates (OBS) and 5 year relative survival rates (REL) of patients with breast and prostatic cancer in Finland in 1970-1981 by hospital district.

\begin{tabular}{|c|c|c|c|c|c|c|c|c|c|}
\hline \multirow{4}{*}{\multicolumn{2}{|c|}{$\begin{array}{l}\text { Hospital } \\
\text { district }^{2}\end{array}$}} & \multicolumn{8}{|c|}{ Primary site } \\
\hline & & \multicolumn{4}{|l|}{ Breast } & \multicolumn{4}{|c|}{ Prostate } \\
\hline & & \multirow[b]{2}{*}{ No } & \multirow[b]{2}{*}{ Age } & \multicolumn{2}{|c|}{ Survival } & \multirow[b]{2}{*}{ No } & \multirow[b]{2}{*}{ Age } & \multicolumn{2}{|c|}{ Survival } \\
\hline & & & & $O B S$ & $R E L$ & & & $\overline{O B S}$ & $R E L$ \\
\hline A & $\begin{array}{l}1 \\
2 \\
3 \\
4\end{array}$ & $\begin{array}{r}4549 \\
1804 \\
674 \\
1562\end{array}$ & $\begin{array}{l}60.0 \\
60.7 \\
60.4 \\
58.1\end{array}$ & $\begin{array}{l}60.9 \\
65.0 \\
60.0 \\
62.1\end{array}$ & $\begin{array}{l}69 \cdot 3 \\
74 \cdot 2 \\
69 \cdot 5 \\
68 \cdot 2\end{array}$ & $\begin{array}{r}1951 \\
1108 \\
326 \\
1005\end{array}$ & $\begin{array}{l}71 \cdot 7 \\
72 \cdot 4 \\
71.8 \\
71.5\end{array}$ & $\begin{array}{l}37 \cdot 1 \\
32 \cdot 8 \\
42 \cdot 2 \\
30 \cdot 6\end{array}$ & $\begin{array}{l}56 \cdot 3 \\
51 \cdot 1 \\
64 \cdot 6 \\
46.9\end{array}$ \\
\hline B & 5 & 808 & $59 \cdot 3$ & $58 \cdot 3$ & $65 \cdot 1$ & 417 & $71 \cdot 4$ & 36.9 & 55.7 \\
\hline C & $\begin{array}{l}6 \\
7 \\
8 \\
9\end{array}$ & $\begin{array}{l}655 \\
410 \\
748 \\
744\end{array}$ & $\begin{array}{l}59 \cdot 7 \\
60.8 \\
58.7 \\
63.7\end{array}$ & $\begin{array}{l}61.6 \\
58.1 \\
59.7 \\
52.8\end{array}$ & $\begin{array}{l}69 \cdot 1 \\
66.3 \\
66.8 \\
61 \cdot 2\end{array}$ & $\begin{array}{l}388 \\
239 \\
486 \\
416\end{array}$ & $\begin{array}{l}71.4 \\
71.9 \\
71.7 \\
71.8\end{array}$ & $\begin{array}{l}35.7 \\
37.2 \\
32.3 \\
31.6\end{array}$ & $\begin{array}{l}54.0 \\
56 \cdot 6 \\
49.1 \\
48 \cdot 1\end{array}$ \\
\hline 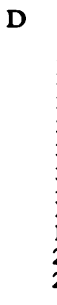 & $\begin{array}{l}10 \\
11 \\
12 \\
13 \\
14 \\
15 \\
16 \\
17 \\
18 \\
19 \\
20 \\
21\end{array}$ & $\begin{array}{r}802 \\
542 \\
674 \\
360 \\
231 \\
461 \\
643 \\
291 \\
210 \\
198 \\
233 \\
79\end{array}$ & $\begin{array}{l}60.0 \\
60.0 \\
58.9 \\
59.9 \\
60.1 \\
59.0 \\
60.6 \\
59.8 \\
58.3 \\
57.7 \\
57.3 \\
62.2\end{array}$ & $\begin{array}{l}56.0 \\
58.4 \\
67.4 \\
61.8 \\
54.1 \\
56.8 \\
55.2 \\
57.2 \\
53.3 \\
62.1 \\
63.7 \\
60.5\end{array}$ & $\begin{array}{l}63.6 \\
66.5 \\
75.8 \\
70.2 \\
60.9 \\
63.5 \\
62.5 \\
64.5 \\
59.4 \\
68.6 \\
69.7 \\
72.4\end{array}$ & $\begin{array}{r}440 \\
307 \\
333 \\
214 \\
139 \\
244 \\
419 \\
183 \\
131 \\
114 \\
152 \\
93\end{array}$ & $\begin{array}{l}71.3 \\
72.4 \\
71.8 \\
72.0 \\
72.0 \\
71.3 \\
72.0 \\
72.1 \\
71.8 \\
73.0 \\
71.6 \\
74.7\end{array}$ & $\begin{array}{l}32.8 \\
35.9 \\
35.9 \\
19.5 \\
28.2 \\
35.2 \\
38.8 \\
34.1 \\
41.3 \\
25 \cdot 7 \\
33.4 \\
28.0\end{array}$ & $\begin{array}{l}49.0 \\
55.4 \\
54.5 \\
30.0 \\
41.8 \\
53.1 \\
59.6 \\
52.2 \\
63.4 \\
42.1 \\
50.1 \\
47.3\end{array}$ \\
\hline \multicolumn{2}{|c|}{ ALL } & 16678 & $60 \cdot 0$ & $60 \cdot 2$ & $68 \cdot 1$ & 9105 & 71.8 & $35 \cdot 3$ & $54 \cdot 0$ \\
\hline
\end{tabular}


distribution than that with standard deviation equal to 1 (several differences $>2$ ) or in a single outlier with an absolute value considerably greater than 2 .

\section{Results}

BREAST CANCER

The total number of breast cancer cases included in the study was 16678 . The number of patients was 79 in the smallest hospital district and 4549 in the largest. The mean age of patients was 60.0 years. The age distribution of patients varied by place of residence. The highest mean age of patients was 63.7 years and the lowest $57 \cdot 3$ years among the 21 hospital districts (table).

The five year observed survival rates varied from $52.8 \%$ to $67.4 \%$. The range of five year relative survival rates was from $59.4 \%$ to $75.8 \%$ (table). Standardised differences of age adjusted five year relative survival rates showed more variation than expected by chance, and the standardised differences for all the university hospital districts with radiotherapy units were positive (fig 1). No such trend was evident for the four other hospital districts equipped with radiotherapy facilities.
The proportion of localised tumours varied from $35 \cdot 6^{\circ}$ o to $63 \cdot 3^{\circ}$ o by district, and that of non-localised from $31.7^{\circ}{ }_{0}$ to $46.9^{\circ}{ }_{0}$. The localised cases and the non-localised cases were considered separately (fig 1). The variation in the survival rates for the non-localised cases exceeded that expected by chance and the standard deviation of the standardised differences was greater than 1. Altogether six (against one expected) differences exceeded the absolute value of 2. For localised cases the standardised differences were less than 2 in absolute value for all districts except one outlier area showing much better survival than in the other hospital districts. Analyses were also made for different age groups separately (not shown), and the pattern of the variation was substantially similar in all age groups.

\section{PROSTATIC CANCER}

The total number of cases with prostatic cancer included in the study was 9105 . The smallest number of patients by hospital district was 93 and the largest 1951. The age distribution of patients varied by place of residence. The highest mean age of patients was 74.7 years and the lowest 71.3 years (table)
Figure 1 Standardised differences of age adjusted 5 year relative survival rates of patients with breast cancer in Finland in 1970-1981 by hospital district and stage.
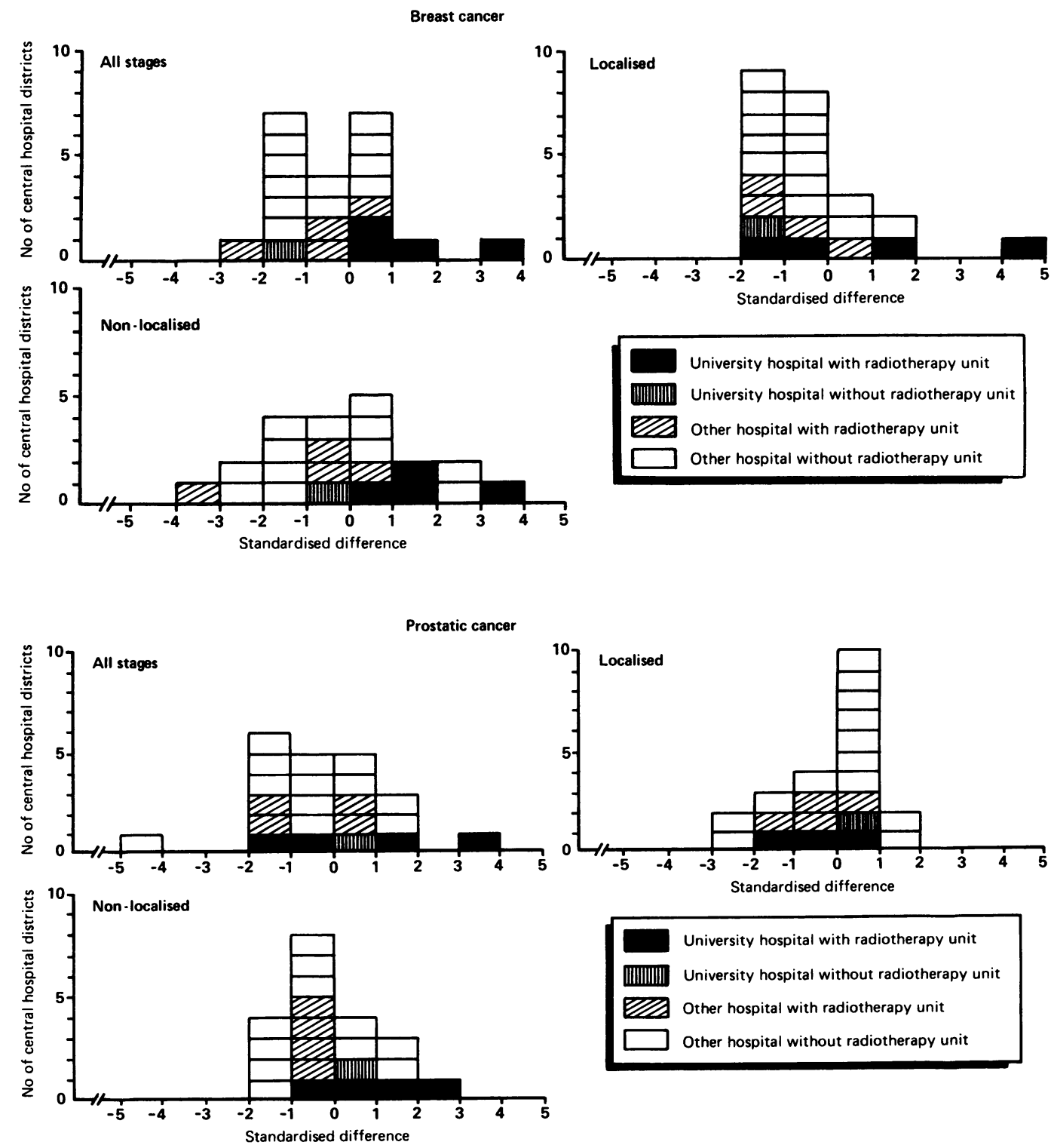

University hospital with radiotherapy unit [|||||||||| University hospital without radiotherapy unit Other hospital with radiotherapy unit

$\square$ Other hospital without radiotherapy unit
Figure 2 Standardised differences of age adjusted rates of patients with prostatic cancer in Finland in 1970-1981 by hospital district and stage. 
The lowest five year observed survival rate was $19.5 \%$ and the highest $42.2 \%$. The five year relative survival rates were between $30.0 \%$ and $64.6 \%$ (table). Two of the standardised differences of age adjusted five year relative survival rates were not between the limits -2 and 2 (fig 2). There was a large difference of -4.3 and 4.0 between these two outliers. The standardised differences were not related to the existence of a university clinic. When survival rates were analysed by the stage of disease at diagnosis, much of the difference for the two outlier districts disappeared (fig 2). There was no indication of variation greater than expected by chance.

\section{Discussion}

Finland is geographically variable: the population density differs by two orders of magnitude from the south to the north, the climate has substantial variations between the north and south, and social structure is correlated with geography. The local municipalities are responsible for the provision of the health services, with expenditure subsidised by the central government. Several municipalities are combined to form a hospital district.

The results were based on 17000 breast cancer patients and on 9000 prostatic cancer patients. Compared to clinical trials on which the routine use and preferences on clinical treatment are based these figures are large indeed. The five year relative survival rates varied from 59 to $76^{\circ}{ }_{0}$ for breast cancer patients and from 30 to $65^{\circ}$ o for prostatic cancer patients. Most of this substantial variation can, however, be accounted for by confounding and by random variation. The method used here for eliminating the confounding effect of age and stage and to evaluate the random variation has been studied earlier and compared with another method based on proportional hazards' model. ${ }^{4}$ It was concluded that with both methods it was possible to detect the systematic variation in survival. Despite the fact that the regression analysis method was more sensitive and more factors could simultaneously be taken into acount, in the present application the method used was also successful in detecting more than random variation.

In the case of breast cancer patients with localised tumours, variation exceeding that expected by chance was due to a single outlier. The distribution of standardised differences was wide for female breast cancer patients with nonlocalised tumours. This pattern showed more variation than expected by chance. For prostatic cancer the apparent systematic variation almost disappeared after adjusting for the obvious confounder of age. In the analysis without stratification by stage, two hospital districts appeared to differ from the others, patients in one district doing very well and patients in the other rather worse.

The more than chance variation in survival for breast cancer patients may be due to incomplete removal of confounding factors or to inequality in health services. There were systematic differences in the type and resources between the hospitals which can be related to the survival of patients residing in the geographical area covered by the hospital district.
The proportion of patients treated by radiation therapy varies between the primary sites considered. ${ }^{712}$ Combined therapy (surgery and postoperative radiotherapy) has been the most frequently used method in breast cancer treatment. Hormonal therapy (including orchiectomy) and radiotherapy without surgery are the most often used methods for prostatic cancer patients. Especially in the case of breast cancer, the type of operation often varies by hospital and even by surgeon.

If, in Finland, a hospital with a cancer unit does not have radiation treatment facilities, the patient is referred for that kind of treatment to the nearest hospital with such equipment, and the overall treatment policy tends to become very similar throughout the country. However, although such referral is common, the selection of radiation treatment for a patient residing in a radiation equipped hospital district is likely to be different from that for a patient residing in an area without its own facilities for radiation treatment. Futhermore, the effect of radiation equipment should be site specific and rather direct for breast cancer, in which radiation treatment is common, and less obvious or indirect for prostatic cancer, where radiation treatment is much less common.

There were only minor differences in survival for breast cancer patients associated with the existence of radiation equipment. This could imply that the organisation functions well and patients are treated by radiation equally effectively irrespective of the local facility for such treatment. Therefore the centralisation of the radiotherapy facilities did not seem to have an effect on the survival of a patient. The second alternative is that radiation treatment does not have any overall effect on survival of patients with breast cancer. It is also possible that the method used is not sensitive enough to detect small differences in survival. ${ }^{4}$

Survival was somewhat better for breast cancer patients resident in university hospital districts. The differences indicate either better treatment due to greater expertise or more indirect effects related to the patients or to the environment. The lead time from first symptoms to diagnostic confirmation may be shorter in university hospital districts due, perhaps, to better diagnostic services and procedures or to better cancer consciousness in the population. This assumes that the crude adjustment by stage into localised and non-localised cases only was not enough to remove all the bias from this source. It is also possible that the results without classification of stage are less subject to bias, because in some areas the metastatic tumours may have mistakenly been classified as localised more often than in some other areas. This would tend to bias the survival rates downwards for both the localised and the non-localised group of patients.

There is also geographical variation in the incidence of cancer in Finland. ${ }^{13}$ People living in the areas with a university hospital are generally of higher socioeconomic status than others and the environmental exposures and personal habits related to cancer risk differ systematically between the university hospital districts and other areas in Finland. If the speed of growth of the tumour is related to the aetiology, then survival 
rates and university hospitals will be spuriously related.

In conclusion, a major part of the large variation in survival of breast cancer patients could be accounted for by chance. There was only minor variation in survival, which could possibly be attributed to health services. The differences in survival were related more to residing in a university hospital district than to residing in a district equipped with a hospital radiotherapy unit. If the differences are due to treatment, the results imply that they are more likely to be due to non-radiation than to radiation treatment. For prostatic cancer the large differences in survival rates of from $30 \%$ to $65 \%$ were due to confounding and to chance, and there was no indication of differences induced by treatment. Therefore, in spite of the large variation in crude survival, there were only minor indications of inequality in cancer treatment in Finland in terms of survival of the patient with breast or prostatic cancer, because most of the variation in survival could be accounted for by chance variation and by confounding of factors not related to health services.
1 Mooney GH. Equity in health care: confronting the confusion. Effective Health Care 1983; 1: 179-85.

2 Montoya-Aguilar C, Marin-Lira MA. Intranational equity in coverage of primary health care: examples from developing countries. WHO Stat $Q$ 1986; 39: 336-44.

3 Musgrove P. Measurement of equity in health. WHO Stat $Q$ 1986; 39: 325-35.

4 Hakama M, Karjalainen S, Hakulinen T. Outcome-based equity in treatment of colon cancer patients in Finland. In f Technology Assessment Health Care 1989; 5: 619-30.

5 Härö S. Health expenditure by area in Finland-an indicator of equity. Health Policy 1987; 7: 299-315.

6 Saxén E, Teppo L. Finnish Cancer Registry. Twenty-five years of a nationwide cancer registry. Helsinki: Finnish Cancer Registry, 1978: 1-47.

7 Hakulinen T, Pukkala E, Hakama M, Lehtonen M, Saxén E, Teppo L. Survival of cancer patients in Finland in 19531974. Ann Clin Res 1981; 13: (suppl 31); 6-7.

8 Cutler SJ, Ederer F. Maximum utilization of the life table method in analyzing survival. $₹$ Chron Dis $1958 ; 8: 699-712$ 9 Ederer F, Axtell LM, Cutler SJ. The relative survival rate: a statistical methodology. Natl Cancer Inst Monogr 1961; 6: 101-21.

10 Hakulinen T. Cancer survival corrected for heterogeneity in patient withdrawal. Biometrics 1982; 38: 933-42.

11 Fleiss JL. Statistical methods for rates and proportions. New York: Wiley, 1981: 240-7.

12 Karjalainen S, Aareleid T, Hakulinen T, Pukkala E, Rahu $M$, Tekkel M. Survival of female breast cancer patients in Finland and in Estonia-stage at diagnosis important determinant of the differences between countries. Soc $S_{C i}$ Med 1989; 28: 233-8.

13 Pukkala E, Gustavsson N, Teppo L. Atlas of cancer incidence in Finland 1953-82. Helsinki: Finnish Cancer Registry
(Cancer Society of Finland publication No. 37), 1987: 1-55. 